Pacific Journal of Mathematics

TENSOR PRODUCTS OF IDEAL SYSTEMS AND THEIR 


\title{
TENSOR PRODUCTS OF IDEAL SYSTEMS AND THEIR MODULES
}

\author{
KARL EgIL AUbert AND ISIDORE Fleischer
}

\begin{abstract}
We prove the existence and furnish an explicit construction of the tensor product in various categories of ideal systems and module systems, structures previously introduced and studied by the first author as a setting for abstract commutative algebra.
\end{abstract}

1. Introduction. Among the various notions of commutative algebra which until recently had not been carried over to the general framework of ideal systems and module systems was the notion of tensor products. In [5] P. Ezust filled this gap for module systems by a fairly laborious categorical approach. He showed namely that the category of module systems (with zero element) over a given ideal system (with zero element) has the requisite properties, including the existence of an appropriate internal Hom-functor in order to secure the presence of a left adjoint to this functor-and hence of the tensor product. In spite of the fact that a direct construction of the tensor product of module systems is to some extent implicit in his work, he makes the comment that it is not clear how such a direct construction might be carried out.

It is the purpose of this paper to give some clarifications and complements to [5] which in particular lead to a direct construction of the tensor product for various categories of module systems and ideal systems. Among these is the tensor product whose existence is established indirectly in [5]. These constructions will be preceded by a general discussion of coinduced ("final") structures for the two basic categories. From the tensor product as constructed in these basic categories, tensor products in more special subcategories are derived by a process of reflection. What gives the present situation a somewhat unusual character is the fact that we are dealing not with purely algebraic systems which are equationally defined, but rather with systems which are similar to combined algebraic-topological structures, like topological monoids.

We would like to thank our good friends Paulo Ribenboim and Larry Cummings as well as Le Centre de Recherches Mathématiques for providing excellent material assistance which enabled us to carry out this work ${ }^{1}$.

${ }^{1}$ Assistance from a Canadian National Research Council operating grant and a Québec-Ontario Exchange Grant is also acknowledged. 
2. Ideal systems and module systems. Let $S$ be a commutative monoid. We shall say that there is defined an ideal system (or $x$ system) $(S, x)$ in $S$ if to every subset $A$ of $S$ there corresponds a subset $A_{x} \subset S$ such that

$$
\begin{gathered}
A \subset A_{x} \\
B \subset A_{x} \rightleftharpoons B_{x} \subset A_{x} \\
S A_{x} \subset A_{x} \\
B A_{x} \subset(B A)_{x} .
\end{gathered}
$$

We say that $A_{x}$ is the $x$-ideal generated by $A$ and in case $A=A_{x}$ we say simply that $A$ is an $x$-ideal. The crucial axiom of the theory is (2.4) which for obvious reasons is referred to as the continuity axiom. An equivalent way of formulating (2.4) is to require that the family $\mathscr{C}$ of all $x$-ideals in $(S, x)$ is closed under the operation of taking residuals:

$$
A_{x} \in \mathscr{X} \Longrightarrow A_{x}: b \in \mathscr{P} \text { for all } b \in S .
$$

In contradistinction to what seems to be a tacit assumption in some earlier papers on ideal systems we do not exclude the possibility $\varnothing \in \mathscr{X}$ which means that the intersection of all the $x$-ideals in $S$ might be void.

By a morphism of ideal systems $f:\left(S_{1}, x_{1}\right) \rightarrow\left(S_{2}, x_{2}\right)$ we mean a mapping of $S_{1}$ into $S_{2}$ such that

$$
\begin{aligned}
& f(a b)=f(a) f(b) \text { for all } a, b \in S_{1} \\
& f\left(A_{x_{1}}\right) \subset(f(A))_{x_{2}} \text { for all } A \subset S_{1} .
\end{aligned}
$$

The condition (2.6) amounts to saying that the inverse image by $f$ of an $x_{2}$-ideal in $S_{2}$ is an $x_{1}$-ideal in $S_{1}$. The category of ideal systems and morphisms of ideal systems will be denoted by IDS.

To define module systems we postulate that the elements of $S$ in an ideal system $(S, x)$ act on a set $M$ which is equipped with a closure operation $U \rightarrow U_{y}(U \subset M)$ such that the following conditions are satisfied

$$
\begin{gathered}
\left.\begin{array}{c}
(a b) u=a(b u) \quad \text { whenever } a, b \in S \text { and } u \in M \\
\qquad U_{y} \subset U_{y} \text { for all } U \subset M \\
A U_{y} \subset(A U)_{y} \\
A_{x} U \subset(A U)_{y}
\end{array}\right\} \text { for all sets } A \subset S, U \subset M .
\end{gathered}
$$

(In the case of an ordinary module over a ring (2.9) and (2.10) are the 
analogues of the two distributive laws one has in such a situation.) If $U=U_{y}$ we say that $U$ is a $y$-module.

We call the whole set-up $(S, x, M, y)$ or more shortly $(M, y)$ - or just $M-a$ module system over the ideal system $(S, x)$ whenever the above requirements are fulfilled. Denoting the family of $x$-ideals in $S$ by $\mathscr{Z}$ and the family of $y$-modules in $M$ by $\mathscr{Y}$ we can give the following equivalent forms of the two continuity axioms (2.9) and (2.10) which will be particularly pertinent in what follows

$$
\begin{gathered}
U_{y} \in \mathscr{Y} \Longrightarrow U_{y}: a \in \mathscr{Y} \text { for all } a \in S \\
U_{y} \in \mathscr{Y} \Longrightarrow U_{y}: u \in \mathscr{X} \text { for all } u \in M .
\end{gathered}
$$

A morphism of module systems is a map $f:\left(M_{1}, y_{1}\right) \rightarrow\left(M_{2}, y_{2}\right)$ such that the following two conditions are satisfied

$$
\begin{gathered}
f(a u)=a f(u) \text { for all } a \in S \text { and all } u \in M_{1} \\
f\left(U_{y_{1}}\right) \subset(f(U))_{y_{2}} \text { for all } U \subset M_{1} .
\end{gathered}
$$

Again (2.12) means that the inverse image of a $y_{2}$-module in $M_{2}$ is a $y_{1}$-module in $M_{1}$. The category of module systems over $(S, x)$ and morphisms of such module systems will be denoted by $\operatorname{MODS}(S, x)$ or simply by MODS when there is no danger of confusion. For a more ample treatment of the fundamentals of the theory of ideal systems and module system one may refer to [1], [2], [3], and [5].

3. Coinduced structures for ideal systems and module systems. The question of coinduced (or "final") structures in a certain category of module systems was treated in [5]. It is desirable, however, to have a more general and fuller treatment of the matter than that presented there.

Let us first look at the category $\operatorname{MODS}(S, x)$ without any restriction on the ideal system $(S, x)$. If in a module system $(S, x, M, y)$ we ignore the closure operations $x$ and $y$ and keep only the action of $S$ on $M$ in accordance with (2.7), we are left with what is called an $S$-set. Any subset $U$ of $M$ such that $S U \subset U$ is called an $S$-set in (or $S$-subset of) $M$. An $S$-set can thus be conceived of as a module system with respect to the generation processes $x_{S}: A \rightarrow S A \cup$ $A(A \subset S)$ in $S$ and $y_{S}: U \rightarrow S U \cup U(U \subset M)$ in $M$ respectively. The morphisms, or $S$-maps, between such systems are those satisfying just (2.11) ((2.12) being a consequence in this case). This provides an jsomorphism between the category of $S$-sets with their $S$-maps and the category of module systems $\left(S, x_{S}, M, y_{S}\right)$.

Proposition 1. Let $\left\{\left(M_{i}, y_{i}\right) \mid i \in I\right\}$ be a family of module systems 
over $(S, x)$ and let $M$ be an $S$-set. Assume further that for each $i \in I$ there is given an $S$-map $g_{i}: M_{i} \rightarrow M$. Then there exists a unique finest closure system $y$ on $M$ such that $(M, y)$ is a module system over $(S, x)$ and such that all the $g_{i}$ 's become morphisms of module systems. The family $\mathscr{Y}$ of all the $y$-modules in $(M, y)$ may be described explicitly as follows: Let $\mathscr{Y}^{*}$ be the family of all those $S$-sets in $M$ which have the property that the inverse image by each $g_{i}$ is a $y_{i}$-module. Then

$$
\mathscr{Y}=\left\{U_{y^{*}} \mid U_{y^{*}} \in \mathscr{V}^{*} \text { and } U_{y^{*}}: m \in \mathscr{Q} \text { for all } m \in M\right\}
$$

where $\mathscr{Z}$ denotes the family of $x$-ideals in $(S, x)$.

The proof of this proposition is largely a routine matter and may be left to the reader. Just one point may deserve special mention, namely that $\mathscr{Y}$ verifies not only the continuity axiom $\left(2.10^{\prime}\right)$ (which is already built into its definition) but also verifies $\left(2.9^{\prime}\right)$. Indeed, let $U_{y} \in \mathscr{Y}$. In order to show that $U_{y}: s \in \mathscr{Y}$ for an $s \in S$ we must show that: (i) $U_{y}: s \in \mathscr{Y}^{*}$ and that (ii) $\left(U_{y}: s\right): m \in \mathscr{P}$ for all $m \in M$. Now $U_{y}: s$ is an $S$-set in $M$, and $g_{i}^{-1}\left(U_{y}: s\right)=g_{i}^{-1}\left(U_{y}\right): s \in \mathscr{Y}_{i}$ for all $i$, which shows (i). The condition (ii) follows from the identity $\left(U_{y}: s\right): m=U_{y}: s m$ together with the definition of $\mathscr{Y}$.

We shall say that $y$ (or $\mathscr{Y}$ ) is coinduced by the $S$-maps $g_{i}$. In certain cases it is not necessary to require explicitly that the sets of $\mathscr{Y}^{*}$ are $S$-sets (i.e., that $S U_{y^{*}} \subset U_{y^{*}}$ for all $U_{y^{*}} \in \mathscr{Y}^{*}$ ), since this condition will automatically be fulfilled if the given family of maps $\left\{g_{i}\right\}$ satisfies a certain covering condition. It should be emphasized, however, that the existence of coinduced structures in the category $\operatorname{MODS}(S, x)$ is not dependent on any such covering condition, a fact which seems to have been overlooked in [5] where the existence of coinduced module systems is made to depend on a very strong covering condition. (It should also be noted that if a covering condition is imposed in order to make all the $U_{y^{*}} \in \mathscr{Y}^{*} S$-sets it would suffice to replace the covering condition given in [5] by the following weaker and more easily applicable one: Any set $U$ in $M$ whose inverse images by the $g_{i}$ 's are $y_{i}$-modules for all $i$ is contained in the union of the images $g_{i}\left(M_{i}\right)$ (and not necessarily in any single $g_{i}\left(M_{i}\right)$ as required in [5]). It is easily seen that this covering condition assures that such a set $U$ is an $S$-set.)

The subject of coinduced ideal systems may be treated analogously and is even simpler than in the case of module systems.

Proposition 2. Let $\left\{\left(S_{i}, x_{i}\right)\right\}$ be a family of ideal systems and let $g_{i}: S_{i} \rightarrow S$ be a family of maps to a commutative monoid $S$ such 
that $g_{i}(a b)=g_{i}(a) g_{i}(b)$ for all $a, b \in S_{i}$ and all $i$. Then there exists a unique finest ideal system $x$ on $S$ making $g_{i}:\left(S_{i}, x_{i}\right) \rightarrow(S, x)$ a morphism of ideal systems for all $i$. The family $\mathscr{X}$ of $x$-ideals in this coinduced ideal system in $S$ is given by

$$
\mathscr{P}=\left\{A_{x^{*}} \mid A_{x^{*}} \in \mathscr{Q}^{*} \text { and } A_{x^{*}}: s \in \mathscr{P}^{*} \text { for all } s \in S\right\}
$$

where $\mathscr{X}^{*}$ is the family of all s-ideats in $S$ whose inverse images by the $g_{i}$ 's are $x_{i}$-ideals for all $i$.

The proof is quite simple and is essentially contained in the proof of Proposition 6 in [1].

4. Tensor products of module systems. In this and the following paragraph we turn to tensor products for module systems and ideal systems, i.e., within the categories $\operatorname{MODS}(S, x)$ and IDS. We construe these tensor products in the usual way as objects which provide a canonical factorization of bimorphisms from $M_{1} \times M_{2}$ : meaning that fixing either of the two arguments at any value $m_{1} \in M_{1}$ or $m_{2} \in M_{2}$ always results in a morphism in the other argument. In the case of module systems over a fixed ideal system $(S, x)$ we consider commutative diagrams of the form

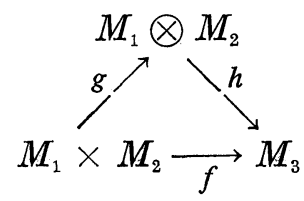

where $M_{1}$ and $M_{2}$ are given, and the task is to determine a unique module system $M_{1} \otimes M_{2}$ equipped with a bimorphism $g$ from $M_{1} \times M_{2}$ to $M_{1} \otimes M_{2}$ such that every bimorphism $f$ from $M_{1} \times M_{2}$ is a composition of the canonical bimorphism $g$ and a unique morphism $h$ from $M_{1} \otimes M_{2}$. In this way all the bimorphisms from $M_{1} \times M_{2}$ are obtained by letting the morphisms from $M_{1} \otimes M_{2}$ "operate on" the fixed bimorphism $g$ - which may thus be considered as a generator of the set of all bimorphisms which have $M_{1} \times M_{2}$ as a domain of definition.

The categories we are dealing with here are of a mixed algebraic topological kind. The algebraic part will be equational so that the tensor product within their poorer structure may be obtained as in the classical case via the free algebra on the (unstructured) set $M_{1} \times M_{2}$ modulo the congruence generated by the minimal identifications which make the quotient map induce a bimorphism of $M_{1} \times M_{2}$ : More precisely it will be this factor algebra equipped with the restriction to $M_{1} \times M_{2}$ of the quotient map as bimorphism. One will be able to convert this algebraic solution into one for the richer mixed 
category whenever the latter has coinduced structures. Indeed the mixed tensor product is then just the algebraic one equipped with the finest closure operation which makes each of the maps obtained by fixing an argument in the canonical bimorphism into a morphism of module systems (or ideal systems as the case may be).

\section{THEOREM 1. The category $\operatorname{MODS}(S, x)$ has tensor products.}

Proof. As already indicated we first consider the module systems $M_{1}$ and $M_{2}$ merely as $S$-sets and take the free $S$-set $\mathscr{F}_{S}\left(M_{1} \times M_{2}\right)$ on the set $M_{1} \times M_{2}$, which will be the disjoint union of copies of $S$ indexed by $M_{1} \times M_{2}$. Instead of using the cumbersome notation $s_{\left(m_{1}, m_{2}\right)}$ for an element in $\mathscr{F}_{S}\left(M_{1} \times M_{2}\right)$ we shall denote this element by $s\left(m_{1}, m_{2}\right)$ and remark that the congruence in question is here generated by the relations

$$
s\left(m_{1}, m_{2}\right)=\left(s m_{1}, m_{2}\right)=\left(m_{1}, s m_{2}\right) .
$$

(Other relations such as $s_{1} s_{2}\left(m_{1}, m_{2}\right)=s_{1}\left(m_{1}, s_{2} m_{2}\right)=\left(s_{1} m_{1}, s_{2} m_{2}\right)$ etc. are easily seen to be derivable from 4.2). Denoting this congruence relation by $\sim$ we put

$$
M_{1} \otimes_{S} M_{2}=\mathscr{F}_{S}\left(M_{1} \times M_{2}\right) / \sim
$$

and let $g: M_{1} \times M_{2} \rightarrow M_{1} \otimes M_{2}$ be the restriction of the canonical quotient map. (This could just as well be obtained as the quotient map for the equivalence generated by (4.2) as in [4] or [9].)

Proposition 1 now tells us how to equip $M_{1} \otimes M_{2}$ with a coinduced structure relative to the union of the two families of $S$-maps $\left\{_{m_{1}} g\right\}$ and $\left\{g_{m_{2}}\right\}$ defined by $m_{1} g(x)=m_{1} \otimes x$ for $m_{1} \in M_{1}$ and $g_{m_{2}}(x)=x \otimes m_{2}$ for $m_{2} \in M_{2}$, thus providing $M_{1} \otimes M_{2}$ with the finest module system making $g$ into a bimorphism. This module system on $M_{1} \otimes M_{2}$ is denoted by $y_{1} \otimes y_{2}$ and the corresponding family of $y_{1} \otimes y_{2}$-modules by $\mathscr{Y}_{1} \otimes \mathscr{Y}_{2}$.

Finally the map $h$ defined by $h\left(m_{1} \otimes m_{2}\right)=f\left(m_{1}, m_{2}\right)$ will now be a morphism of module systems making the diagram (4.1) commutative. Clearly, $h$ is a well-defined $S$-map. To show that $h$ is also a morphism it will thus suffice to show that $h^{-1}\left(U_{y_{3}}\right) \in \mathscr{Y}_{1} \otimes \mathscr{Y}_{2}$ whenever $U_{y_{3}} \in \mathscr{Y}_{3}$. First of all $h^{-1}\left(U_{y_{3}}\right)$ is an $S$-set since $h$ is an $S$-map and $U_{y_{3}}$ is an $S$-set. Using the notation ${ }_{m_{1}} f$ and $f_{m_{2}}$ analogously to ${ }_{m_{1}} g$ and $g_{m_{2}}$ above, we have ${ }_{m_{1}} f=h \circ{ }_{m_{1}} g$ and $f_{m_{2}}=h \circ g_{m_{2}}$. Since $f$ is a bimorphism ${ }_{m_{1}} f$ and $f_{m_{2}}$ are morphisms of module systems i.e.,

$$
{ }_{m_{1}} g^{-1}\left(h^{-1}\left(U_{y_{3}}\right)\right) \in \mathscr{Y}_{2} \text { and } g_{m_{2}}^{-1}\left(h^{-1}\left(U_{y_{3}}\right)\right) \in \mathscr{Y}_{1} \text { for all } m_{1}, m_{2} .
$$

Taking into account that the structure on $M_{1} \otimes M_{2}$ is the coinduced 
one, this means that $h^{-1}\left(U_{y_{3}}\right)$ belongs to the family which corresponds to $\mathscr{Y}^{*}$ in Proposition 1. But $h^{-1}\left(U_{y_{3}}\right)$ also belongs to the "cut-down" family (designated by $\mathscr{Y}$ in Proposition 1) since $h^{-1}\left(U_{y_{3}}\right): m \in \mathscr{Q}$ for all $m=m_{1} \otimes m_{2} \in M_{1} \otimes M_{2}$. Indeed, from $f_{m_{2}}^{-1}\left(U_{y_{3}}\right) \in \mathscr{Y}_{1}$ for $U_{y_{3}} \in \mathscr{Y}_{3}$ it follows that $f_{m_{2}}^{-1}\left(U_{y_{3}}\right): m_{1} \in \mathscr{Q}$ for all $m_{1} \in M_{1}$, and since $f_{m_{2}}=h \circ g_{m_{2}}$ this means that $g_{m_{2}}^{-1}\left(h^{-1}\left(U_{y_{3}}\right)\right): m_{1}=h^{-1}\left(U_{y_{3}}\right): g_{m_{2}}\left(m_{1}\right)=h^{-1}\left(U_{y_{3}}\right):\left(m_{1} \otimes m_{2}\right) \epsilon$ $\mathscr{Q}$ for all $m_{1} \otimes m_{2} \in M_{1} \otimes M_{2}$. This completes the proof of Theorem 1 .

5. Tensor products of ideal systems. In the present paragraph we prove

TheOREM 2. The category of ideal systems has tensor products.

Proof. Let $\left(S_{1}, x_{1}\right)$ and $\left(S_{2}, x_{2}\right)$ be two ideal systems. In line with our general approach we first look for a monoid bimorphism $g$ which factors every bimorphism $f$ from $S_{1} \times S_{2}$ the other factor being a unique monoid morphism $h$ from $S_{1} \otimes S_{2}$ as in the following diagram.

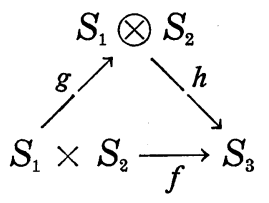

That $f$ is a bimorphism means that

$$
\begin{aligned}
& f\left(s_{1}, s_{2} t_{2}\right)=f\left(s_{1}, s_{2}\right) f\left(s_{1}, t_{2}\right) \text { and } \\
& f\left(s_{1} t_{1}, s_{2}\right)=f\left(s_{1}, s_{2}\right) f\left(t_{1}, s_{2}\right)
\end{aligned}
$$

for all $s_{1}, t_{1}$ in $S_{1}$ and $s_{2}, t_{2}$ in $S_{2}$. We first form the free commutative monoid $\mathscr{F}\left(S_{1} \times S_{2}\right)$. In accordance with (5.2) one considers the congruence relation $\sim$ generated by the two relations $\left(s_{1}, s_{2} t_{2}\right)=$ $\left(s_{1}, s_{2}\right)\left(s_{1}, t_{2}\right)$ and $\left(s_{1} t_{1}, s_{2}\right)=\left(s_{1}, s_{2}\right)\left(t_{1}, s_{2}\right)$ and puts

$$
S_{1} \otimes S_{2}=\mathscr{F}\left(S_{1} \times S_{2}\right) / \sim \text {. }
$$

(See also [6] and [7].)

In complete analogy with the previous development for module systems, consider the coinduced structure on $S_{1} \otimes S_{2}$ which results

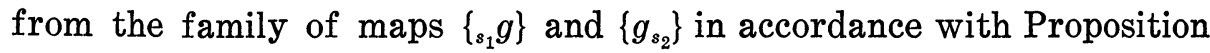
2. This defines an ideal system $x_{1} \otimes x_{2}$ on $S_{1} \otimes S_{2}$ with a corresponding family of $x_{1} \otimes x_{2}$-ideals denoted by $\mathscr{X}_{1} \otimes \mathscr{X}_{2}$.

It is again easy to see that map $h$ defined by $h\left(s_{1} \otimes s_{2}\right)=f\left(s_{1}, s_{2}\right)$ will be a morphism of ideal systems from $\left(S_{1} \otimes S_{2}, x_{1} \otimes x_{2}\right)$ into $\left(S_{3}, x_{3}\right)$. Since $h$ is already a multiplicative (monoid) morphism we need only 
verify that $h^{-1}\left(A_{x_{3}}\right) \in \mathscr{Z}_{1} \otimes \mathscr{Z}_{2}$ for every $A_{x_{3}} \in \mathscr{Z}_{3}$ - which means according to Proposition 2 that the following conditions hold for all $s_{1}, t_{1} \in S_{1}, s_{2}, t_{2} \in S_{2}$ and all $A_{x_{3}} \in \mathscr{Z}_{3}$.

$$
\begin{gathered}
h^{-1}\left(A_{x_{3}}\right) \text { is an } s \text {-ideal in } S_{1} \otimes S_{2} \\
g_{s_{2}}^{-1}\left(h^{-1}\left(A_{x_{3}}\right)\right) \in \mathscr{Z}_{1} \text { and }{ }_{s_{1}} g^{-1}\left(h^{-1}\left(A_{x_{3}}\right)\right) \in \mathscr{Z}_{2} \\
g_{s_{2}}^{-1}\left(h^{-1}\left(A_{x_{3}}\right):\left(t_{1} \otimes t_{2}\right)\right) \in \mathscr{X}_{1} \text { and } \\
{ }_{s_{1}} g^{-1}\left(h^{-1}\left(A_{x_{3}}\right):\left(t_{1} \otimes t_{2}\right)\right) \in \mathscr{X}_{2} .
\end{gathered}
$$

To prove (5.3) let $b \in h^{-1}\left(A_{x_{3}}\right)$, hence $h(b) \in A_{x_{3}}$ and $h(s) h(b) \in A_{x_{3}}$ for all $s \in S_{1} \otimes S_{2}$. Thus $h(s b) \in A_{x_{3}}$ and $s b \in h^{-1}\left(A_{x_{3}}\right)$ as required. The verification of (5.4) is similar to the verification of (4.3) above and may be left to the reader. Finally (5.5) results easily by using the identity $h^{-1}\left(A_{x_{3}}\right):\left(t_{1} \otimes t_{2}\right)=h^{-1}\left(A_{x_{3}}: h\left(t_{1} \otimes t_{2}\right)\right)$ together with ${ }_{s_{1}} f=h_{\circ_{s_{1}}} g$ and $f_{s_{2}}=h \circ g_{s_{2}}$.

6. Tensor products in certain subcategories of IDS and $\operatorname{MODS}(S, x)$. Many of the ideal systems and module system which have been considered in the literature, e.g., those mentioned in [1], [2], and [3], are more restricted than those we have chosen here for the comprehensive categories IDS and $\operatorname{MODS}(S, x)$ in that they require the presence of zero elements. However, the notion of a zero generalizes slightly differently in the context of ideal systems and that of module systems.

A multiplicative zero in a commutative monoid $S$ is an element $0 \in S$ such that $a 0=0$ for all $a \in S$. Such a multiplicative zero in $S$, if it exists, is unique.

If $(S, x)$ is an ideal system we shall say that the element $0 \in S$ is an $x$-zero if $\{0\}_{x}=\{0\}$. If 0 is an $x$-zero it is also a multiplicative zero. Any nonvoid $x$-ideal in an ideal system with a multiplicative zero will contain this zero element and the possibility $\varnothing \in \mathscr{Z}$ may safely be dispensed with as uninteresting. Hence we postulate that the presence of a zero rules out the possibility that the void set be counted as an $x$-ideal. We define the $x$-kernel of an ideal system $(S, x)$ as the $x$-ideal $A_{x}^{0}$ which is the intersection of all the $x$-ideals in $S$ : thus the smallest $x$-ideal in $(S, x)$. According to the above convention $A_{x}^{0} \neq \varnothing$ whenever $(S, x)$ has a multiplicative zero 0 , and then $A_{x}^{0}=\{0\}_{x}$.

The notion which in the "module" situation parallels that of a multiplicative zero is that' of an element $\theta$ in an $S$-set $M$ such that $a \theta=\theta$ for all $a \in S$. If $(M, y)$ is a module system over $(S, x)$ then $\theta$ is said to be a $y^{*}$-zero if $\{\theta\}_{y}=\{\theta\}$. Again $a \theta=\theta$ for all $a \in S$ when $\theta$ is a $y^{*}$-zero. Whereas an $x$-zero is uniquely determined by 
the requirement $\{0\}_{x}=\{0\}$ the corresponding requirement $\{\theta\}_{y}=\{\theta\}$ does not determine $\theta$ uniquely in $M$. In order to obtain uniqueness it is reasonable to define a $y$-zero in $M$ as an element $\theta$ such that $\{\theta\}$ is equal to the intersection of all the nonvoid $y$-modules in $(M, y)$. On the other hand if $S$ has a multiplicative zero 0 such that $0 m=\theta$ this requirement will of itself impose unicity on $\theta$. In the case of module systems also we agree to discard the void set as a $y$-module in the presence of a $y$-zero. Furthermore the $y$-kernel $U_{y}^{0}$ of $(M, y)$ is defined as the intersection of all the $y$-modules in $(M, y)$.

Let IDS $_{0}$ denote the category of ideal systems with an $x$-zero and morphisms of ideal systems. Correspondingly $\operatorname{MODS}_{0}(S, x)$ will denote the category of module systems with a $y$-zero over $(S, x)$ and morphisms of module systems. The categories $\operatorname{IDS}_{0}$ and $\operatorname{MODS}_{0}(S, x)$ sit as full subcategories in IDS and $\operatorname{MODS}(S, x)$ respectively. In order to construct the tensor products in $\operatorname{IDS}_{0}$ and $\operatorname{MODS}_{0}(S, x)$ we shall employ the notion of a Rees-congruence and the corresponding formation of factor systems. By means of this we can show that $\operatorname{IDS}_{0}$ and $\operatorname{MODS}_{0}(S, x)$ are reflective subcategories in $\operatorname{IDS}$ and $\operatorname{MODS}(S, x)$ respectively and this categorical fact will tell us how to obtain the tensor product in the smaller category when we know it in the bigger. We shall return briefly to this general categorical viewpoint after we have treated the special cases of $\operatorname{IDS}_{0}$ and $\operatorname{MODS}_{0}(S, x)$.

Given an ideal ( $s$-ideal) in the monoid $S$ (i.e., a subset $A_{s}$ of $S$ such that $S A_{s} \subset A_{s}$ ) the Rees-congruence modulo $A_{s}$ is defined by declaring any two elements in $A_{s}$ as congruent to each other whereas any element outside of $A_{s}$ is only congruent to itself. A similar definition applies also in the "module" situation modulo $S$-subsets instead of $s$-ideals. In particular this applies to an $x$-ideal $A_{x}$ in an ideal system $(S, x)$ and to a $y$-module $U_{y}$ in a module system $(M, y)$. Denoting the factor systems modulo the Rees-congruence by a double bar, we obtain an ideal system $\left(S / / A_{x}, \overline{\bar{x}}\right)$ and a module system $\left(M / / U_{y}, \bar{y}\right)$ by imposing the finest systems $\bar{x}$ and $\bar{y}$ which make the canonical quotient maps into morphisms (by Propositions 1 and 2 for a single $i$ ).

THEOREM 3. The categories $\operatorname{MODS}_{0}(S, x)$ and $\operatorname{IDS}_{0}$ have tensor products.

Proof. We give the proof only in the case of $\operatorname{MODS}_{0}(S, x)$, the proof for $\mathrm{IDS}_{0}$ being quite similar. We first note that the full subcategory $\operatorname{MODS}_{*}(S, x)$ consisting of those module systems for which the $y$-kernel is nonvoid is closed under the taking of tensor products in $\operatorname{MODS}(S, x)$. This follows from the definition of the tensor product together with the fact that $\operatorname{MODS}_{*}(S, x)$ is closed for 
coinduced structures since the inverse image of the void set is void. Moreover, this subcategory contains $\operatorname{MODS}_{0}(S, x)$. The advantage of restricting ourselves to the subcategory $\mathrm{MODS}_{*}$ is that the Rees factor system modulo the $y$-kernel in this subcategory will always yield a module system with a $y$-zero. Thus if we first form the tensor product in $\operatorname{MODS}(S, x)$ of systems $M_{1}, M_{2}$ in $\operatorname{MODS}_{0}(S, x)$ and then pass to the Rees quotient modulo its nonvoid $y$-kernel $U_{y_{1} \otimes y_{2}}^{0}$, we obtain in $M_{1} \otimes M_{2} / / U_{y_{1} \otimes y_{2}}^{0}$ an object of $\operatorname{MODS}_{0}(S, x)$. This gives rise to the following extension of the Diagram (4.1) where also $M_{3}$ is now supposed to be in $\operatorname{MODS}_{0}(S, x)$.

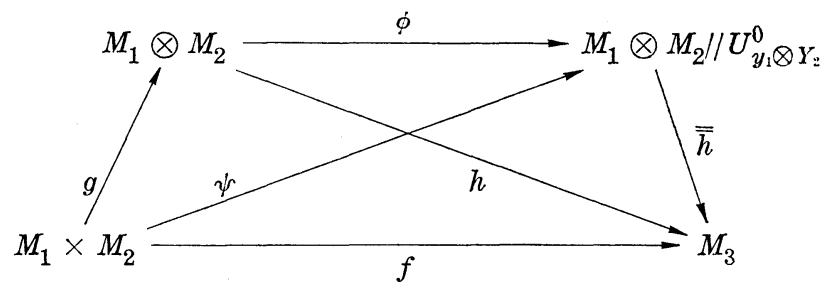

Here $\phi$ is the canonical quotient morphism, $\psi=\phi \circ g$ and $\overline{\bar{h}}$ is defined by $\left.\overline{\bar{h}} \overline{\overline{m_{1} \otimes m_{2}}}\right)=h\left(m_{1} \otimes m_{2}\right)\left(=f\left(m_{1}, m_{2}\right)\right)$ where $\overline{\overline{m_{1} \otimes m_{2}}}$ denotes the Rees residue class to which $m_{1} \otimes m_{2}$ belongs modulo the $y_{1} \otimes y_{2}$-kernel. The map $\overline{\bar{h}}$ is well-defined because of the definition of the Reescongruence together with the fact that $U_{y_{1} \otimes y_{2}}^{0} \subset h^{-1}\left(\theta_{3}\right), h$ being a morphism and $M_{3}$ having a $y_{3}$-zero $\theta_{3}$. Finally one easily verifies that $\overline{\bar{h}}$ is a morphism taking into account that the Rees factor system modulo $U_{y_{1} \otimes y_{2}}^{0}$ is coinduced by $\phi$.

From the unique factorization $f=\overline{\bar{h}} \circ \psi$ it is clear that the bimorphism $\psi$ solves the universal problem for bimorphisms in the category $\operatorname{MODS}_{0}(S, x)$ and establishes the module system

$$
\left(M_{1} \otimes M_{2} / / U_{y_{1} \otimes y_{2}}^{0}, \overline{\overline{y_{1} \otimes y_{2}}}\right)
$$

as the tensor product of $\left(M_{1}, y_{1}\right)$ and $\left(M_{2}, y_{2}\right)$ in the category $\operatorname{MODS}_{0}(S, x)$.

The Diagram (6.1) just represents the conjunction of the solutions to two different universal problems. Whereas the lower left triangle consisting of the arrows $f, g$, and $h$ gives the canonical factorization of bimorphisms in $\operatorname{MODS}(s, x)$ the upper right triangle consisting of the arrows $h, \phi$, and $\overline{\bar{h}}$ gives the canonical factorization of a morphism which goes into an object in $\operatorname{MODS}_{0}(S, x)$ : Any morphism of the latter kind can be uniquely factored through the Rees factor system.

7. Tensor products in reflective subcategories. A subcategory $\mathscr{B}$ of $\mathscr{A}$ is said to be reflective in $\mathscr{A}$ [8] when the inclusion functor $\mathscr{B} \rightarrow \mathscr{A}$ has a left adjoint. This left adjoint functor $R$ is sometimes 
called a reflector and the adjoint functor $\mathscr{A} \rightarrow \mathscr{B}$ a reflection of $\mathscr{A}$ in its subcategory $\mathscr{B}$. A reflection may be described in terms of universal morphisms: $\mathscr{B} \subset \mathscr{A}$ is reflective if and only if to each $A \in \mathscr{A}$ there is an object $R A$ of the subcategory $\mathscr{B}$ and a morphism in $\mathscr{A} g_{A}: A \rightarrow R A$ such that every morphism $f: A \rightarrow B$ where $B$ is in $\mathscr{B}$ has the form $f=h \circ g_{A}$ for a unique morphism $h: R A \rightarrow B$ of $\mathscr{B}$. What we have made use of in constructing the tensor product in $\operatorname{MODS}_{0}(S, x)$ is that it is a reflective subcategory of $\operatorname{MODS}_{*}(S, x)$ with the functor $R:(M, y) \rightarrow\left(M / / U_{y}^{0}, y\right)$ as reflector. In general, the tensor product in any reflective subcategory $\mathscr{B}$ of a concrete category $\mathscr{A}$ may be obtained from the tensor product in $\mathscr{A}$ according to the formula

$$
B_{1} \bigotimes_{\mathscr{B}} B_{2}=R\left(B_{1} \bigotimes_{\mathscr{A}} B_{2}\right)
$$

As a further illustration of this procedure consider the subcategory $\operatorname{MODSEP}_{0}(S, x)$ consisting of module systems over $(S, x)$ with a separating $y$-zero. According to [2] and [5] a module system $(M, y)$ is said to have a separating $y$-zero $\theta$ if $\theta$ is a $y$-zero and $\left(m_{1}\right)_{y}=$ $\left(m_{2}\right)_{y} \Rightarrow m_{1}=m_{2}\left(m_{1}, m_{2} \in M\right)$. A $y$-zero may be made separating, and more generally a Rees factor system may be reduced to a MODSEP system, by dividing out by a strengthened congruence: Given a $y$-module $U_{y}$ in $(M, y)$ one introduces as in [3] a $y$-congruence modulo $U_{y}$ by putting $u \equiv v\left(U_{y}\right)$ whenever $\left(U_{y}, u\right)_{y}=\left(U_{y}, v\right)_{y}$. The resulting factor module system $(\bar{M}, \bar{y})=\left(M / U_{y}, \bar{y}\right)$ over $(S, x)$ where $\bar{y}$ is the finest module system in $\bar{M}$ making the canonical map $(M, y) \rightarrow(\bar{M}, \bar{y})$

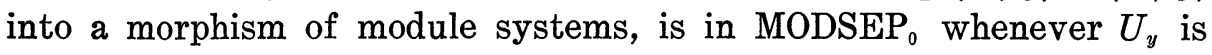
nonvoid. In fact, that the $y$-zero $\theta$ is separating amounts to saying that the $y$-congruence modulo $\theta$ reduces to equality. Thus $\operatorname{MODSEP}_{0}(S, x)$ is reflective subcategory of $\operatorname{MODS}_{*}(S, x)$ with $R:(M, y) \rightarrow$ $\left(M / U_{y}^{0}, \bar{y}\right)$ as reflector. It is also possible to view $\operatorname{MODSEP}_{0}(S, x)$ as a reflective subcategory of $\operatorname{MODS}_{0}(S, x)$ with $(M, y) \rightarrow\left(M /(\theta)_{y}, \bar{y}\right)$ as reflector. In either case the tensor product in $\operatorname{MODSEP}_{0}(S, x)$ may be obtained from the general formula (7.1). It goes without saying that the same procedure applies to the corresponding category IDSEP in the case of ideal systems. In [6] this procedure has been used implicitly to construct tensor products of commutative semigroups as well as tensor products of semigroups with zero from an initial construction of tensor products for general (i.e., noncommutative) semigroups.

\section{REFERENCES}

1. K. E. Aubert, Theory of x-ideals, Acta Mathematica, 107 (1962), 1-52.

2. —_ Additive ideal systems, J. Algebra, 18 (1971), 511-528. 
3. K. E. Aubert and E. R. Hansén, Systèmes de modules, C. R. Acad. Sci. Paris, 272 (1971), 525-528.

4. M. Delorme, Sur la platitude des demi-groupes de fractions, C. R. Acad. Sci. Paris, 269 (1969), 630-632.

5. P. Ezust, On a representation theory for ideal systems, forthcoming paper in Pacific J. Math.

6. P. A. Grillet, The tensor product of semigroups, Trans. Amer. Math. Soc., 138 (1969), 267-280 and 281-293.

7. T. J. Head, Homomorphisms of commutative semigroups as tensor maps, J. of Nat. Sci. and Math., 7 (1967), 39-49.

8. S. MacLane, Categories for the Working Mathematician, N.Y., Springer-Verlag, 1971.

9. B. Stenström, Flatness and localization over monoids, Math. Nachrichten, 48 (1971), 315-334.

Received February 24, 1977 and in revised form September 12, 1977.

UNIVERSITET I OSLO

POSTBOKS 1053

Blindern, OSLO 3, NoRway

AND

Centre de Recherches Math.

UNIVERSITÉ DE MONTRÉAL

C. P. 6128

Montréal, H3C 3J7, Canada 


\section{PACIFIC JOURNAL OF MATHEMATICS}

\section{EDITORS}

RICHARD ARENS (Managing Editor)

University of California

Los Angeles, CA 90024

Charles W. Curtis

University of Oregon

Eugene, OR 97403

C. C. Moore

University of California

Berkeley, CA 94720

\section{J. DugundJI}

Department of Mathematics

University of Southern California

Los Angeles, CA 90007

R. FInN and J. Milgram

Stanford University

Stanford, CA 94305

\section{ASSOCIATE EDITORS}
E. F. BECKENBACH
B. H. NeumanN
F. WOLF
K. YoSHIDA

\section{SUPPORTING INSTITUTIONS}

\author{
UNIVERSITY OF BRITISH COLUMBIA \\ CALIFORNIA INSTITUTE OF TECHNOLOGY \\ UNIVERSITY OF CALIFORNIA \\ MONTANA STATE UNIVERSITY \\ UNIVERSITY OF NEVADA, RENO \\ NEW MEXICO STATE UNIVERSITY \\ OREGON STATE UNIVERSITY \\ UNIVERSITY OF OREGON
}

\author{
UNIVERSITY OF SOUTHERN CALIFORNIA \\ STANFORD UNIVERSITY \\ UNIVERSITY OF HAWAII \\ UNIVERSITY OF TOKYO \\ UNIVERSITY OF UTAH \\ WASHINGTON STATE UNIVERSITY \\ UNIVERSITY OF WASHINGTON
}

The Supporting Institutions listed above contribute to the cost of publication of this Journal, but they are not owners or publishers and have no responsibility for its content or policies.

Mathematical papers intended for publication in the Pacific Journal of Mathematics should be in typed form or offset-reproduced, (not dittoed), double spaced with large margins. Please do not use built up fractions in the text of the manuscript. However, you may use them in the displayed equations. Underline Greek letters in red, German in green, and script in blue. The first paragraph or two must be capable of being used separately as a synopsis of the entire paper. Items of the bibliography should not be cited there unless absolutely necessary, in which case they must be identified by author and journal, rather than by item number. Manuscripts, in triplicate, may be sent to any one of the editors. Please classify according to the scheme of Math. Reviews, Index to Vol. 39. All other communications should be addressed to the managing editor, or Elaine Barth, University of California, Los Angeles, California, 90024.

50 reprints to each author are provided free for each article, only if page charges have been substantially paid. Additional copies may be obtained at cost in multiples of 50 .

The Pacific Journal of Mathematics is issued monthly as of January 1966. Regular subscription rate: $\$ 72.00$ a year (6 Vols., 12 issues). Special rate: $\$ 36.00$ a year to individual members of supporting institutions.

Subscriptions, orders for numbers issued in the last three calendar years, and changes of address should be sent to Pacific Journal of Mathematics, 103 Highland Boulevard, Berkeley, California, 94708. Older back numbers obtainable from Kraus Periodicals Co., Route 100, Millwood, NY 10546.

PUBLISHED BY PACIFIC JOURNAL OF MATHEMATICS, A NON-PROFIT CORPORATION

Printed at Kokusai Bunken Insatsusha (International Academic Printing Co., Ltd.). 8-8, 3-chome, Takadanobaba, Shinjuku-ku, Tokyo 160, Japan.

Copyright (C) 1978 by Pacific Journal of Mathematics

Manufactured and first issued in Japan 


\section{Pacific Journal of Mathematics

Vol. 74, No. $2 \quad$ June, 1978

Aharon Atzmon, Spectral synthesis in some spaces of bounded continuous

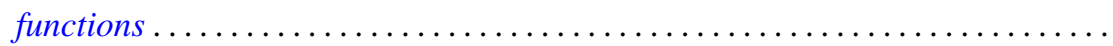

Karl Egil Aubert and Isidor Fleischer, Tensor products of ideal systems and their modules.............................................

Richard F. Basener, Several dimensional properties of the spectrum of a uniform

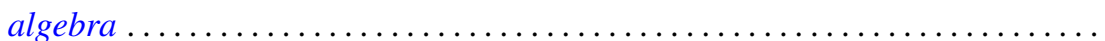

R. H. Bing and Michael Peter Starbird, Super triangulations ............. 307

Andrew Carson, Coherent polynomial rings over regular rings of finite index .....

Robert M. DeVos and Frederick W. Hartmann, Sequences of bounded summability domains .................................................

George Grätzer and R. Padmanabhan, Symmetric difference in abelian groups ....

Robert L. Griess, Jr., A remark about groups of characteristic 2-type and

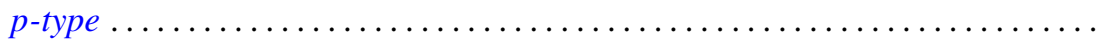

Emil Grosswald and F. J. Schnitzer, A class of modified $\zeta$ and L-functions........

Jutta Hausen and Johnny Albert Johnson, Ideals and radicals of some

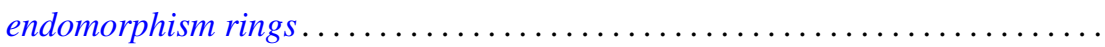

Jean Ann Larson, A solution for scattered order types of a problem of Hagendorf ............................................

Peter A. McCoy, Extremal properties of real biaxially symmetric potentials in $E^{2(\alpha+\beta+2)}$

Héctor Alfredo Merklen, Hereditary crossed product orders .

Hal G. Moore and Adil Mohamed Yaqub, Equational definability of addition in certain rings...

Robert Laurens Moore, Reductivity in $C^{*}$-algebras and essentially reductive operators. . .

Joseph Alvin Neisendorfer, Lie algebras, coalgebras and rational homotopy theory for nilpotent spaces...

William Raymond Nico, Bounded monoids

Richard Paul Osborne, Simplifying spines of 3-manifolds ...

Richard Paul Osborne, The simplest closed 3-manifolds. With an appendix by Osborne and J. Yelle.

Clayton Collier Sherman, The $K$-theory of an equicharacteristic discrete valuation ring injects into the $K$-theory of its field of quotients.... .

Mitchell Herbert Taibleson, The failure of even conjugate characterizations of $H^{1}$

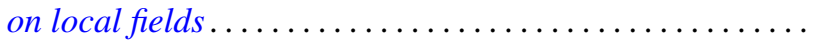

Keti Tenenblat, On characteristic hypersurfaces of submanifolds in Euclidean space ...................................

Jeffrey L. Tollefson, Involutions of Seifert fiber spaces..........

Joel Larry Weiner, An inequality involving the length, curvature, and torsions of a curve in Euclidean $n$-space .......................

Neyamat Zaheer, On generalized polars of the product of abstract homogeneous polynomials.... 